\title{
Matricellular Protein Expression and Cell Ultrastructure as Parameters to Test In Vitro Cytotoxicity of a Biomimetic Scaffold
}

Sandra Durante ${ }^{1}$, Gabriella Teti ${ }^{1}$, Viviana Salvatore ${ }^{1}$, Stefano Focaroli ${ }^{1}$, Monica Mattioli-Belmonte $^{2}$, Concetta Ferretti ${ }^{2}$, Adriana Bigi $^{3}$, Roberto Giardino ${ }^{4}$ and Mirella Falconi* ${ }^{1 *}$

${ }^{1}$ Department for Biomedical and Neuromotor Sciences (DIBINEM), University of Bologna, via Irnerio 48, Bologna, 40126 Italy

${ }^{2}$ Department of Clinical and Molecular Sciences, School of Medicine, Università Politecnica delle Marche, Via Tronto 10/60126, Ancona, Italy

${ }^{3}$ Department of Chemistry "G. Ciamician", University of Bologna, via Selmi 2, 40128 Bologna Italy

${ }^{4}$ BITTA Laboratory, Rizzoli Orthopedic Institute, Via di Barbiano 1/10, Bologna, 40136, Italy

\begin{abstract}
Following scaffold implantation, cell sufferance, in-vivo encapsulation, foreign body reaction and inflammatory response has been reported and the up- regulation of matricellular proteins is often connected with this condition.

Cytotoxicity of biomaterials is generally tested according to ISO standard 10993-5 based mainly on viability tests. Additional assays, based on improved cytotoxicity knowledge, are suggested in order to better analyze the biocompatibility of implant materials.

The purpose of the study was to evaluate the matricellular protein expression as biomarker for in vitro-testing the biocompatibility of implant materials. Tenascin- $C$, osteocalcin and osteopontin belong to the matricellular protein family and were chosen as cytotoxicity markers.

Mesenchymal stem cells were seeded on collagen/hydroxyapatite scaffold and on carboxymethyl cellulose based hydrogel in order to evaluate gene/protein expression by cell viability test, Real Time PCR and western blot. Electron microscopy was carried out to evaluate the morphological changes induced by cell/scaffold interactions.

A low expression of tenascin-c, osteonectin and osteopontin was demonstrated in collagen/hydroxyapatite scaffold compared to the cells cultured on tissue flasks and on hydrogel scaffold.

Based on our results, we propose matricellular protein expression as parameter for testing in vitro biocompatibility of implant materials.
\end{abstract}

Keywords: Matricellular proteins; In vitro biocompatibility; Biomaterials; Mesenchymal stem cells; Real Time PCR

\section{Introduction}

Cytotoxicity assays are widely used in in-vitro biocompatibility studies. Currently, the in vitro cytotoxicity of implant materials is being tested according to ISO standard 10993-5 [1], based mainly on viability tests. The lactate dehydrogenase ( $\mathrm{LDH})$ leakage, the protein assay, the neutral red and the MTT assays are those most commonly employed in the detection of cytotoxicity or cell viability following exposure to biomaterials [2]. In vitro macrophage responses are widely utilized for inflammation reaction [3]. A different sensitivity was observed for each assay with the neutral red and MTT assays being the most sensitive in detecting cytotoxic events as compared to the LDH leakage and the protein assays. Although viability assays can be used to predict the compatibility of medical devices [4], the necessity of more sensitive tests which investigate gene and protein expression in cells grown in contact with biomaterials is strongly recommended in order to predict the behaviour of a implant materials before in vivo testing.

The inflammatory response, biological encapsulation and foreign body reaction, and an unusual reaction to injury occurring in vivo after the implantation of the biomaterials can impair the performance of implanted scaffolds [5]. Key molecules which have a role in modulating the in vivo reaction to the implantation of materials have recently been explored and are referred to as matricellular proteins [6]. The matricellular proteins are a group of extracellular matrix (ECM) proteins which are synthesized by a number of different cell types including fibroblasts, macrophages and endothelial cells [7]. They can regulate cell movement, proliferation and differentiation during morphogenesis and the inflammatory and reparative response following tissue injury and regeneration. The expanding family of matricellular proteins includes, osteonectin (SPARC), osteopontin (OPN) and tenascin-C (TNC) [7].

Abundantly expressed during development, their level is generally low under steady state conditions in adult tissue, but they are readily upregulated in wound healing and tissue remodelling [8-10], conditions which activate an inflammatory response. An aberrant expression of matricellular proteins is often detected in tumors where they actively contribute to tumor progression and metastasis [11].

The aim of the study was to propose matricellular protein expression, such as TNC, SPARC and OPN, as a potential assay in testing the in vitro biocompatibility of implant materials. The idea was to propose a more in vitro sensitive assay for predicting the behaviour of biomaterials in inducing adverse reactions after in vivo implantation. Our hypothesis was that elevated expression of matricellular proteins in cells grown on implant materials was the key signal for potential encapsulation, foreign body reaction and the inflammatory response which could strongly impair the efficiency of the material [12]. On the

*Corresponding author: Mirella Falconi, Department of Biomedical and Neuromotor Sciences (DIBINEM), University of Bologna, via Irnerio, 48-40126 Bologna (Italy), Tel: +39 051 2091511; Fax: +39 051 251735; E-mail: mirella.falconi@unibo.it

Received June 03, 2014; Accepted July 11, 2014; Published July 13, 2014

Citation: Durante S, Teti G, Salvatore V, Focaroli S, Mattioli-Belmonte M, et al. (2014) Matricellular Protein Expression and Cell Ultrastructure as Parameters to Test In Vitro Cytotoxicity of a Biomimetic Scaffold. J Cytol Histol 5: 262. doi:10.4172/2157-7099.1000262

Copyright: (C) 2014 Durante S, et al. This is an open-access article distributed under the terms of the Creative Commons Attribution License, which permits unrestricted use, distribution, and reproduction in any medium, provided the original author and source are credited. 
contrary, a low expression of matricellular proteins could be connected to an elevated biocompatibility of the material.

Mesenchymal stem cells (MSCs) seeded on a gelatin/hydroxyapatite scaffold, widely used in the orthopaedic field for bone regeneration [13] and grown for different periods of time ranging from 7 to 28 days, were utilized for the study. To investigate the influence of the pure material on cell behaviour, MSCs were not exposed to any osteogenic medium, to avoid an in vitro differentiation. SPARC, OPN and TNC are also connected with osteogenic differention. In our model, avoiding the in vitro osteogenesis, we expected that a high expression of matricellular proteins connected with osteogenesis is eliminated.

An MTT assay was performed to test the toxicity of material while ultrastructural morphological changes of cells in contact with the surface of the biomaterial were investigated by transmission electron microscopy (TEM). The mRNA expression of TNC, SPARC and OPN were analyzed using Quantitative Real-Time-Polymerase Chain Reaction (qRT-PCR) and the protein expression was assayed by western blot analysis. All the results were compared to MSCs seeded on plastic dishes (monolayer system). To simulate cellular sufferance conditions we treated the MSCs with hydrogen peroxide, a well-known inductor of stress oxidation and apoptosis. This condition induced an upregulation of matricellular protein expression. Finally, we tested the mRNA expression of TNC, SPARC and OPN on hydrogel scaffolds based of carboxymethyl cellulose, which appeared to have a low biocompatibility in some our previous experiments and from literature [14].

\section{Materials and Methods}

\section{Periodontal ligament mesenchymal stem cell (PDL-MSC) isolation}

Human PDL-MSCs were obtained from 10 healthy volunteers ranging from 16-30 years of age after informed consent was obtained. The tissues were carefully minced and collected in $60 \times 15 \mathrm{~mm}$ cellcultured dishes in Dulbecco's Modified Eagle Medium / F12 (DMEM/ F12) (Gibco, Life Technologies, Monza, Italy) supplemented with $10 \%$ Fetal Bovine Serum (FBS, Gibco, Life Technologies, Monza, Italy) and $1 \%$ penicillin/streptomycin, and were then incubated at $37^{\circ} \mathrm{C}$ in a humidified atmosphere of $5 \% \mathrm{CO}_{2}$.

After 20 days of culture, numerous cells, forming colonies, migrated from the explants. The cells were subcultured once a week using $1 \%$ trypsin (Gibco), expanded in new T75 flasks and maintained at $37^{\circ} \mathrm{C}$ in a humidified atmosphere of $5 \% \mathrm{CO}_{2}$. Cells from passages 2 to 5 were utilized for the experiments described.

\section{Flow cytometric analysis}

According to The International Society for Cellular Therapy guidelines [15] at the end of the $3^{\text {rd }}$ passage, PDL-MSCs were analyzed using a FACSCalibur flow cytometry system (Becton Dickinson, CA, USA) and subjected to differentiation into mesenchymal lineages.

For immunophenotyping, in agreement with minimal criteria for the identification of human MSCs, $2.5 \times 10^{5}$ cells were removed with D-PBS and were then stained for $45 \mathrm{~min}$ with the following antibodies:fluorescein isothiocyanate-(FITC)-labeled mouse antihuman CD90 (StemCell Technologies- Milan, Italy), CD105, CD14, CD19 (Diaclone, France), R-phycoerythrin-(PE)-labeled mouse anti human CD34, CD44, CD45 (Diaclone, France), CD73 (Becton Dickinson, CA, USA) and anti HLA-DR purchased from Diaclone.

The control for FITC- or PE-coupled antibodies was isotypic mouse IgG1. The data were evaluated using CellQuest software (Becton Dickinson, CA, USA).

\section{Gelatin/hydroxyapatite scaffold}

The gelatin/genipin scaffold synthesis and characterization has previously been described in Panzavolta and colleagues [13]. Briefly, hydroxyapatite (HA) $10 \mathrm{wt} \%$ (wt\% of HA with respect to the total weight of HA and gelatin), were added to $140 \mathrm{~mL}$ of a gelatin solution. The suspensions were maintained under constant mechanical stirring $(\sim 700 \mathrm{rpm})$ at $55^{\circ} \mathrm{C}$. After $40 \mathrm{~min}$ of stirring, $10 \mathrm{~mL}$ of an aqueous solution containing $0.234 \mathrm{~g}$ of genipin (Wako, Japan), were added to the suspensions in order to crosslink the gelatin. The gelatin concentration in the final volume was $10 \mathrm{wt} \%$. Following the addition of genipin, the foam assumed a blue color as a result of the binding with the primary amino groups of the gelatin. After approximately $20 \mathrm{~min}$, the suspension was deposited onto Petri dishes. The samples were allowed to gelify in an oven at $55^{\circ} \mathrm{C}$ for $2 \mathrm{~h}$. The samples were then washed in $0.1 \mathrm{M}$ glycine aqueous solution for $30 \mathrm{~min}$ and were then rinsed in distilled water for $5 \mathrm{~min}$. Finally, the samples were frozen in liquid $\mathrm{N}_{2}$ for up to $10 \mathrm{~min}$ and then freeze-dried for $24 \mathrm{~h}$ at $-44^{\circ} \mathrm{C}$ and $0.3 \mathrm{mbar}$.

\section{Cell seeding on a gelatin scaffold}

The MSCs were seeded into gelatin/HA scaffolds and in T75 tissue flasks at a density of $5 \times 10^{6}$ cells $/ \mathrm{ml}$. After 20 minutes at $37^{\circ} \mathrm{C}$, the medium was replaced with fresh medium and the scaffolds were maintained in a humidified atmosphere at $37^{\circ} \mathrm{C}$ with $5 \% \mathrm{CO}_{2}$ in air. The medium was changed every 3-4 days.

For the experiments, the MSCs seeded on the gelatin/HA scaffold and into plastic T75 flasks were collected after 7, 14, 21 and 28 days.

\section{Cellular stress induction}

The MSCs were seeded at a density of $5 \times 10^{5} \mathrm{cells} / \mathrm{ml}$ in DMEM/ F12 supplemented with $10 \%$ FBS and $1 \%$ penicillin/streptomycin and were then incubated at $37^{\circ} \mathrm{C}$ in a humidified atmosphere of $5 \% \mathrm{CO}_{2}$. To simulate stress conditions in growing cells and verify the elevated level of extracellular matrix protein expression, the MSC cells were treated with $0.5 \mathrm{mM} \mathrm{H}_{2} \mathrm{O}_{2}$, for $3 \mathrm{~h}$ in a humidified atmosphere at $37^{\circ} \mathrm{C}$ with $5 \% \mathrm{CO} 2$ in air. Data showed the average of triplicates \pm SD and were representative from three independent experiments

\section{MTT assay}

The MSCs $\left(1 \times 10^{4}\right)$ were seeded onto gelatin scaffolds in 96 multi wells with DMEM/F12 supplemented with $10 \%$ FBS, $1 \%$ penicillin/ streptomycin, and were then incubated at $37^{\circ} \mathrm{C}$ in a humidified atmosphere of $5 \% \mathrm{CO}_{2}$ for $1,3,5$, and 7 days. Subsequently, the samples were incubated with fresh medium containing $0.5 \mathrm{mg} / \mathrm{mL}$ of 3-(4,5-dimethylthiazol-2-yl)-2,5-diphenyltetrazolium bromide (MTT) for $2 \mathrm{~h}$ at $37^{\circ} \mathrm{C}$. The formazan produced was dissolved using a solvent solution ( $0.1 \mathrm{~N} \mathrm{HCl}$ in isopropanol), and the optical density was read at $570 \mathrm{~nm}$ by Microplate Reader (Model 680, Biorad LabInc., CA, USA). Data showed the average of triplicates \pm SD and were representative from three independent experiments.

\section{Transmission Electron Microscopy (TEM)}

At each experimental time, the samples were fixed with $1 \%$ glutaraldehyde in $0.1 \mathrm{M} \mathrm{PB}$ for 2 hours at $4^{\circ} \mathrm{C}$ and were subsequently post-fixed with $1 \% \mathrm{OsO}_{4}$ in $0.1 \mathrm{M} \mathrm{PB}$ for 1 hour at room temperature (RT). After several washes, the samples were dehydrated in an ascending alcohol series $(25 \%, 50 \%, 70 \%, 90 \%, 100 \%)$ and embedded in LR white 
resin (Fluka, Sigma Aldrich, St. Louis, Missouri, USA). Thin sections were collected on nickel grids, stained with uranyl acetate and lead citrate, and were observed using CM10 Philips TEM (FEI Company, Eindhoven, The Netherlands).

The images were recorded using a Megaview III digital camera (FEI Company, Eindhoven, The Netherlands). The images of Figure 3 were representative from two independent experiments.

\section{Quantitative Real Time Polymerase Chain Reaction (qRT- PCR)}

Total RNA was extracted in MSCs treated and untreated with $\mathrm{H}_{2} \mathrm{O}_{2}$ and in MSCs grown on gelatin/HA scaffolds for 7, 14, 21 and 28 days, by NucleoSpin RNA I kit (Machery-Nagel, Duren, Germany), quantified using a NanoDrop ND-1000 UV-Vis Spectrophotometer (Thermo Scientific, Wilmington, DE, USA), and cDNA was transcribed with reverse transcriptase SUPIII (Invitrogen, Carlsbad, CA, USA). The expression of mRNA was analyzed by quantitative Real Time PCR using 7500 Real Time PCR (Applied Biosystem, Life Technologies, Monza, Italy). For the analysis, the following TaqMan assays (Applied Biosystems, Life Technologies, Monza, Italy) were used: osteonectin (Hs00234160_m1), osteopontin (SPP1 Hs00959010_m1), and tenascin-C (Hs01115665_m1). The relative gene expressions were normalized to glyceraldehyde 3-phosphate dehydrogenase (GAPDH Hs99999905_m1), and the data were presented as the fold change using the formula $2^{-\triangle \Delta \mathrm{CT}}$ as recommended by the manufacturer (User Bulletin No.2 P/N 4303859, Applied Biosystems). Data showed the average of triplicates \pm SD and were representative from three independent experiments.

As controls MSCs were seeded at a density of $5 \times 10^{6}$ cells $/ \mathrm{ml}$ on carboxymethyl cellulose based hydrogel (Sigma Aldrich, St Louis, Missouri USA) and in tissue flasks and were grown for 7, 14, 21 and 28 days. At the end of each experimental point, total RNA was extracted as previously described.

\section{Protein extraction and western blot analysis}

MSCs cultured on gelatin/HA scaffolds as previously described, were lysed for 30 minutes using a cell extraction buffer (Invitrogen, Life Technologies, Monza Italy) supplemented with a protease inhibitor cocktail (Sigma Aldrich, St Louis, Missouri, USA), $1 \mathrm{mM}$ phenylmethylsulfonyl fluoride (PMSF) and $0.15 \%$ betamercaptoethanol (Fluka, Sigma Aldrick, St Louis, Missouri, USA). The samples were centrifuged at $14,000 \mathrm{rpm}$ for 10 minutes a $4^{\circ} \mathrm{C}$, and the total protein amounts were assayed using Breadford reagent (Sigma Aldrich, St. Louis, Missouri, USA).

Thirty $\mu$ g of total protein were resolved on $\mathrm{NuPAGE}^{\oplus}$ SDS-PAGE precast Gels (4-12\%) (Invitrogen, Life Technologies, Monza, Italy), and the protein was transferred to a nitrocellulose membrane (GE Healthcare Europe $\mathrm{GmbH}$, Milan, Italy), blocked with $2.5 \%$ bovine serum albumin (BSA) $/ 2.5 \%$ no fat dry milk (Sigma Aldrich, St. Louis, Missouri, USA), and immunolabeled with anti-SPARC 1:100 in blocking reagent (Santa Cruz Biotechnologies, Santa Cruz, CA, USA), anti-osteopontin 1:100 in blocking reagent (Santa Cruz, Biotechnology, Santa Cruz, CA, USA), anti-tenascin-C 1:100 in blocking reagent (Santa Cruz, Biotechnology, Santa Cruz, CA, USA), and anti-GAPDH 1:10000 in blocking reagent (Sigma Aldrich, St. Louis, Missouri, USA). For each reaction, the specific horseradish peroxidase-conjugated antibody was utilized as a secondary antibody for $2 \mathrm{~h} 30 \mathrm{~min}$ at RT.

The bands were visualized by an ECL Advanced TM Western blotting detection kit (GE Healthcare Europe GmbH, Milan, Italy), and the images were recorded using a Kodak digital image station (Eastman Kodak, Rochester, NY, USA). Images utilized are representative from three independent experiments.

Band densitometry was measured using Image J software (National Institutes of Health), and the intensities of the specific protein bands were corrected for equal glyceraldehyde 3-phosphate dehydrogenase loading; they were expressed as relative to the intensity of the $7 \mathrm{D}$ sample. Data showed the average of triplicates \pm SD and were representative from three independent experiments

\section{Statistical analysis}

Statistical analysis was carried out using GRAPH PAD PRISM 5.0 software (San Diego, CA, USA) by applying ANOVA and the Dunnett's multiple comparison test for MTT and band densitometry data, and by applying ANOVA and the Tukey's multiple comparison test for the qRT-PCR data. The differences were considered significant at $\mathrm{p}<0.05$.

\section{Results}

\section{Flow cytometry (FACS) analysis of MSCs}

The MSCs utilized for all additional experiments were characterized for CD105, CD44, CD14, CD19, CD34, CD45, CD73, CD90 and HLADR using flow cytometric analysis. It was found that these cells were highly positive for CD105, CD44, CD90 and CD73, and negative for CD14, CD19, CD34, HLA-DR and CD45 (Figure 1).

\section{MTT assay}

To evaluate whether the gelatin/HA scaffold induced cytotoxic effects on the MSCs seeded on it, an MTT assay was carried out. The results showed an increase of viability in the MSCs during the time of culture (Figure 2), demonstrating elevated biocompatibility of the material. The assay was performed from one day of cell culture to 7 days.

\section{TEM analysis of the MSCs grown on gelatin/HA scaffolds}

The MSC cells were seeded on gelatin/HA for a period of time ranging from 7 to 28 days. After 7 days of culture, the cells showed high adhesion to the scaffold surface, suggesting elevated biocompatibility of the material (Figure 3A). A light deposition of extracellular matrix was detected (Figure 3B) after 14 days of culture while it was more evident at the end of 21 days. The cells showed cytoplasm with several secretive vacuoles (Figure $3 \mathrm{C}$ and insert), suggesting notable production of the extracellular matrix components [16]. After 28 days of growing on the scaffold, thick fibrilles in the extracellular matrix were well observed (Figure 3D), showing a clear banding structure resembling fibers of collagen type I proteins (Figure 3E). The cells grown in monolayer for 28 days showed a disorganized extracellular matrix in which small fibers without any banding structure and with random orientation were detected (Figure 3F) [17].

\section{Cellular stress induction}

To simulate cellular sufferance conditions, we treated the MSCs with $0.5 \mathrm{mM}$ hydrogen peroxide, a well-known inductor of oxidative stress and apoptosis, for $3 \mathrm{~h}$ [18]. The expression of OPN, SPARC and TNC was analyzed in the MSCs exposed to $\mathrm{H}_{2} \mathrm{O}_{2}$ to verify whether stress conditions enhanced the expression of the matricellular proteins. The results showed a significant increase in expression of all the three markers analyzed (Figure 4), supporting our hypothesis. 
Citation: Durante S, Teti G, Salvatore V, Focaroli S, Mattioli-Belmonte M, et al. (2014) Matricellular Protein Expression and Cell Ultrastructure as Parameters to Test In Vitro Cytotoxicity of a Biomimetic Scaffold. J Cytol Histol 5: 262. doi:10.4172/2157-7099.1000262
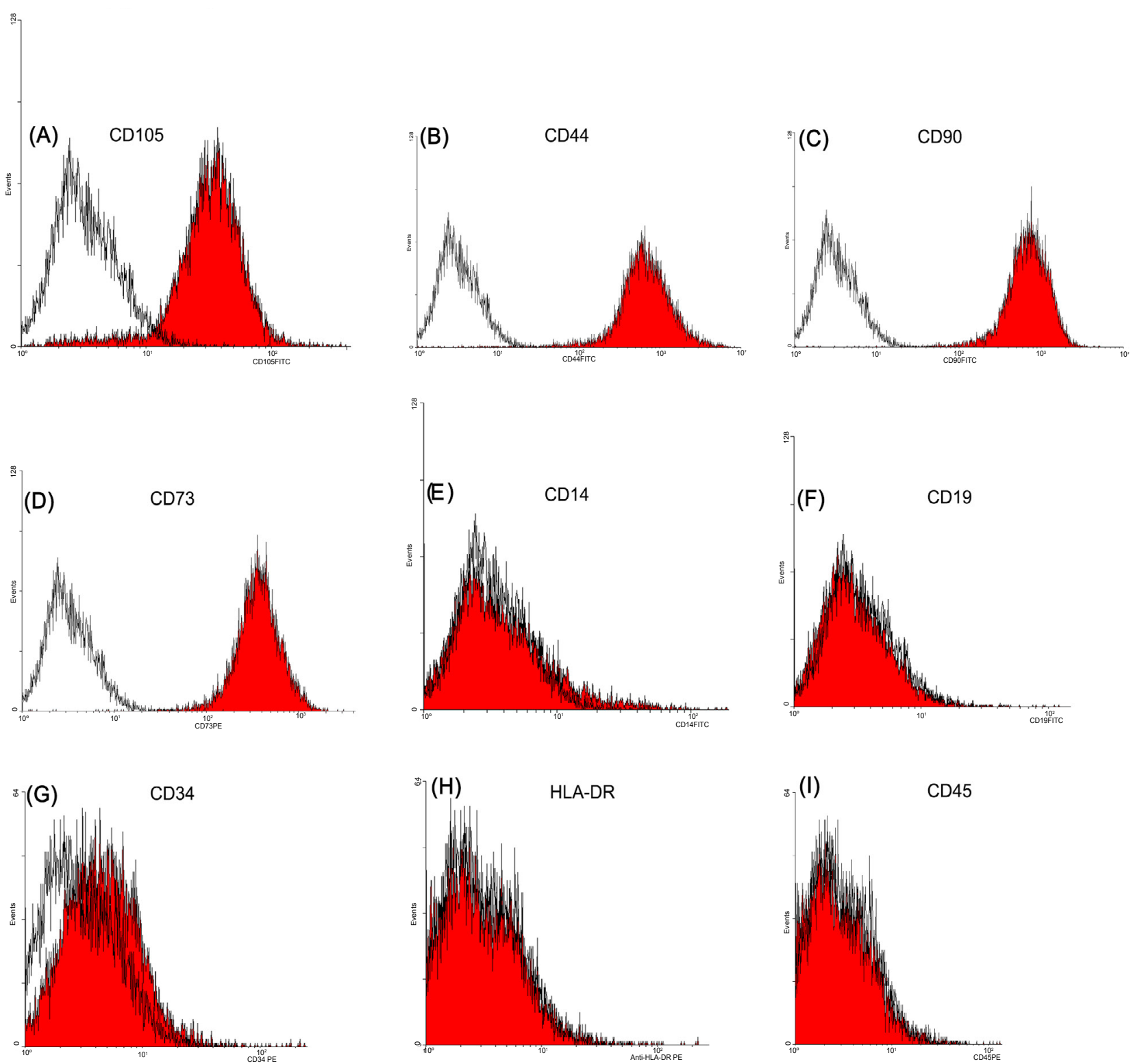

Figure 1: PDLMSC phenotypic profile. Cells showing a positive reaction for CD105, CD44, CD90 and CD73 (A-D), and a negative reaction for CD14, CD19, CD34, $\mathrm{HLA}-\mathrm{DR}$ and CD45 (E-I). Histograms are representative from three independent experiments.

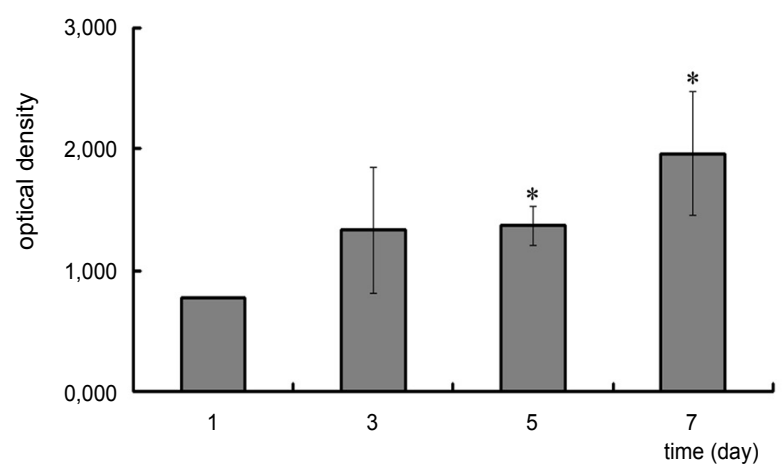

Figure 2: Cell viability assay of MSCs grown on a gelatin/hydroxyapatite scaffold. Data represent results from three independent experiments. Each individual assay was performed in triplicates and expressed as mean of percentage \pm SD. * represents a significant difference as compared to MSCs cultured for 1 day. 
Citation: Durante S, Teti G, Salvatore V, Focaroli S, Mattioli-Belmonte M, et al. (2014) Matricellular Protein Expression and Cell Ultrastructure as Parameters to Test In Vitro Cytotoxicity of a Biomimetic Scaffold. J Cytol Histol 5: 262. doi:10.4172/2157-7099.1000262

Page 5 of 8

\section{qRT-PCR of Tenascin-C, Osteonectin and Osteopontin in MSCs grown on gelatin/HA and carboxymethyl hydrogel scaffolds}

The mRNA expression of matricellular proteins, such as TNC, SPARC and OPN, was evaluated by qRT-PCR in cells grown on gelatin/

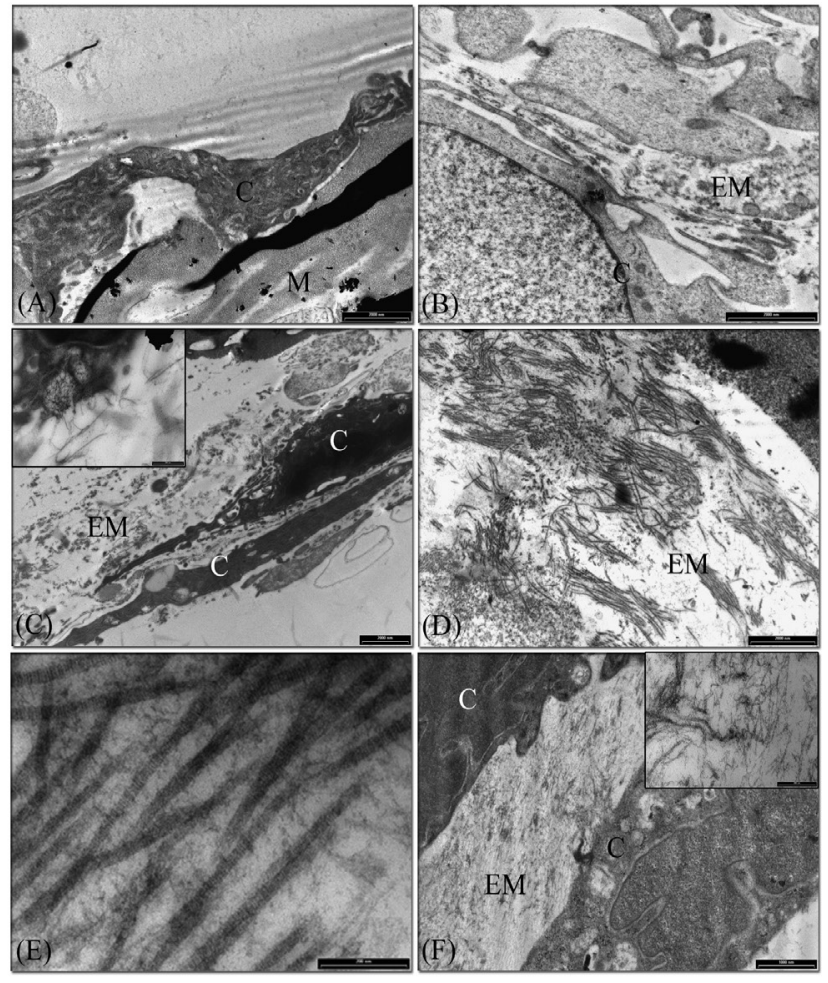

Abbreviation: (C) MSCs; (M) 3D material; (EM) extracellular matrix

Figure 3: TEM images of MSCs cultured on 3D scaffold for (A) 7 days. The cells showed a prolonged body strictly adhering to the scaffold surface (bar: $2 \mu \mathrm{m}$ ); (B) MSCs after 14 days of 3D culture. (bar: $2 \mu \mathrm{m}$ ); (C) MSCs after 21 days of 3D culture. The cells showed several secretive vacuoles (insert) (bar: 2um; insert bar: $500 \mathrm{~nm}$ ); (D) MSCs after 28 days of 3D culture. An elevated deposition of extracellular matrix is shown (bar: $2 \mu \mathrm{m}$ ); (E) high magnification of extracellular matrix in MSCs grown on a 3D scaffold for 28 days. Fibers with a banding structure are clearly detected (bar: $200 \mathrm{~nm}$ ); (F) TEM images of MSCs grown on 2D culture system for 28 days. A lower deposition of extracellula matrix is detected (bar: $1 \mu \mathrm{m})$. High magnification of the organization of extracellular matrix (insert; bar: $200 \mathrm{~nm}$ ).

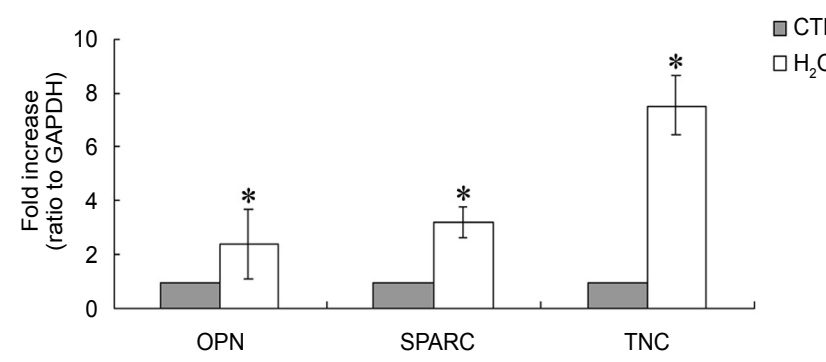

Abbreviations: (OPN) osteopontin; (SPARC) osteonectin; (TNC) tenascin-C

Figure 4: Expression of OPN, SPARC and TNC mRNA in MSCs treated with $0.5 \mathrm{mM}$ hydrogen peroxide. Data represent results from three independent experiments. Each individual assay was performed in triplicates and expressed as mean $\pm \mathrm{SD}$; ${ }^{*} \mathrm{P}<0.05$ compared untreated cells (CTR).

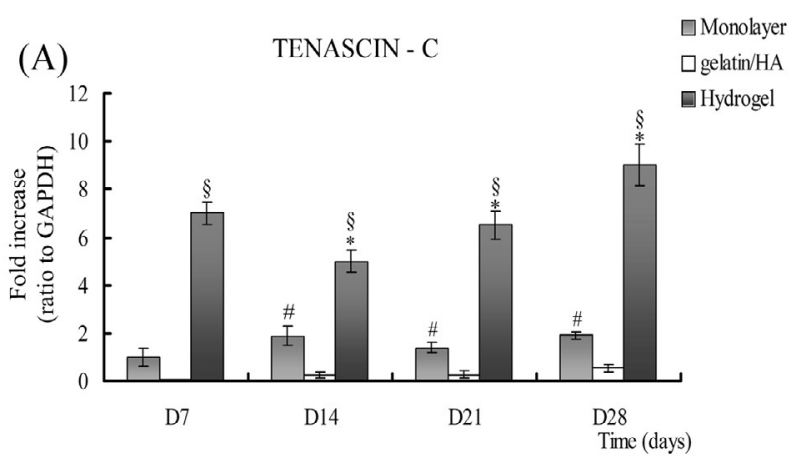

(B)

SPARC

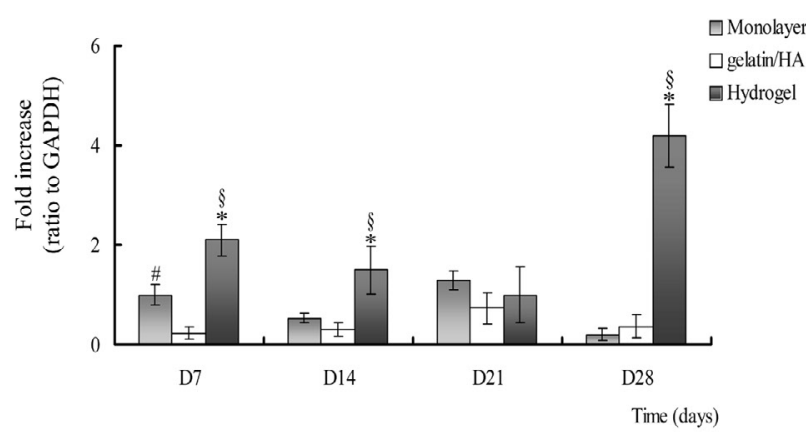

(C)

OSTEOPONTIN

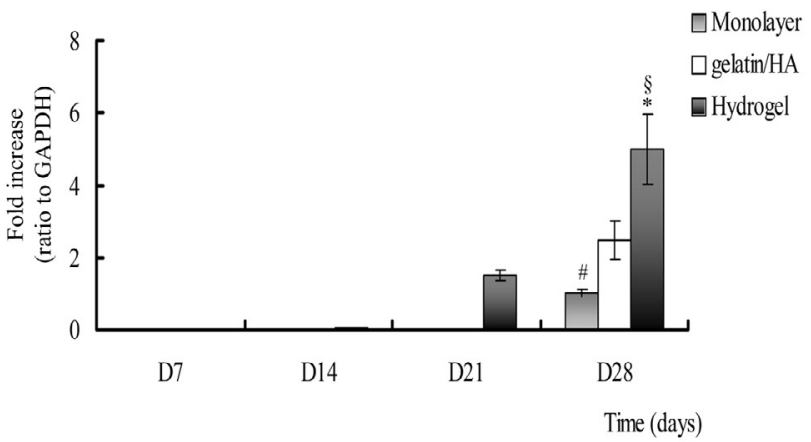

Abbreviations: (TNC) tenascin-C; (SPARC) osteonectin; (OPN) osteopontin Figure 5: (A) Expression of TNC mRNA in MSCs cultured on a gelatin/HA culture system as compared to a monolayer system and hydrogel scaffold. (B) Expression of SPARC mRNA in MSCs cultured on a gelatin/HA culture system as compared to a monolayer system and hydrogel scaffold. (C) Expression of OPN mRNA in MSCs cultured on a gelatin/HA culture system as compared to a monolayer system and hydrogel scaffold. Data represent results from three independent experiments. Each individual assay was performed in triplicates and expressed as mean $\pm S D$; \# represents a significant difference of a gelatin/ $\mathrm{HA}$ culture from monolayer, $\mathrm{P}<0.05$; ${ }^{*}$ represents a significant difference of a gelatine/HA from hydrogel, $\mathrm{P}<0.05$; $\S$ represents a significant difference of hydrogel from monolayer, $\mathrm{P}<0.05$.

HA scaffolds, and the results were compared to the same cells grown in monolayer and on carboxymethyl hydrogel based scaffold.

The analysis of matricellular proteins is based on qRT-PCR which represents a very sensitive method and an improvement in the knowledge regarding the toxicity and biocompatibility of implant materials analyzed according to ISO standard 10993-5, based mainly on viability tests.

Expression of TNC, SPARC and OPN mRNA in the MSCs cultured 
on gelatin/HA scaffolds was significantly lower as compared to cells cultured in monolayer on tissue flasks (Figure 5). On the contrary the expression of TNC, SPARC and OPN mRNA in the MSCs cultured on hydrogel scaffold was significantly higher compared to cells seeded in monolayer and MSCs cultured on gelatin/HA scaffold (Figure 5).

\section{Western blot analysis of TNC, SPARC and OPN proteins in MSCs grown on gelatin/HA scaffolds}

Western blot analysis showed that the protein expression of TNC, SPARC and OPN resembled the mRNA expression as previously described. Tenascin-C had an elevated signal after 21 days of culture on gelatin/HA (Figure 6A-B) while SPARC and OPN showed an elevated signal after 28 days of cultured on scaffold (Figure 6A-B), confirmed by quantitative analysis. The cells grown on monolayer showed a very low level of all the proteins, as already described by qRT-PCR.

\section{Discussion}

The aim of the study was to propose matricellular protein expression, such as TNC, SPARC and OPN, as a potential assay in testing the cytotoxicity of implant materials. The idea was to suggest a more in vitro biochemical and biomolecular sensitive assay for predicting the behaviour of materials in inducing adverse reactions after in vivo implantation.

The cellular model utilized consists of adult MSCs, widely utilized in regenerative medicine and tissue engineering due to their ability to self-renew and differentiate into multiple tissues, including bone, cartilage, fat and other tissues of mesenchymal origin. These properties make them successful candidates for clinical applications $[16,19,20]$. Flow cytometry characterization demonstrated that the MSCs utilized in our study, is in agreement with the criteria of International Society for Cellular Therapy [15].

As implant material a gelatin/hydroxyapatite scaffold was tested due to the wide utilization in the orthopedic field for bone regeneration [13]. Gelatin is a denatured collagen commercially available as a biodegradable polymer. It has been extensively utilized for pharmaceutical and medical purposes, and its biosafety has been proven with long clinical applications [21]. The addition of inorganic components, such as hydroxyapatite enhances both osteoconductivity and osteoinductivity $[13,22]$. Although gelatine scaffolds are considered highly biocompatible, the utilization of genipin as a crosslinking agent could potentially impair the material due to the release of toxic molecules [13]. MTT assay was performed from 1 day to 7 days, in according

(A)

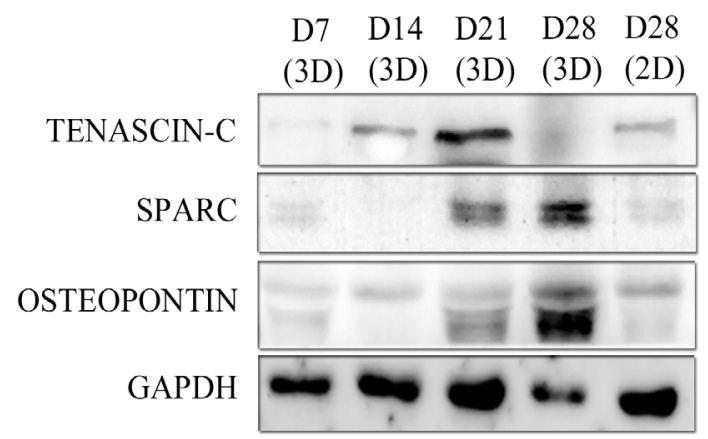

(B)
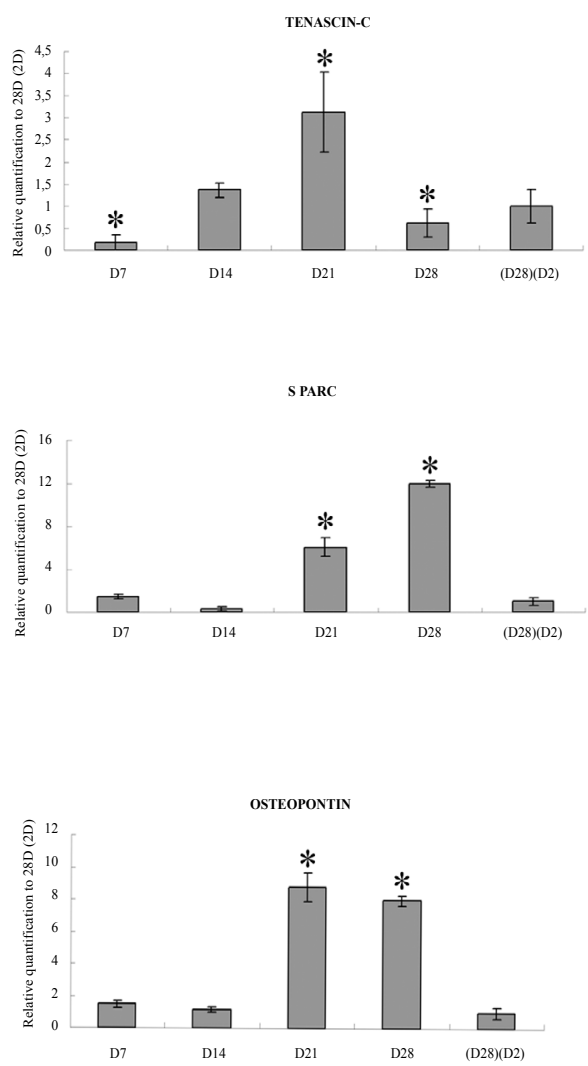

Abbreviations: (TNC) tenascin-C; (SPARC) osteonectin; (OPN) osteopontin; (GAPDH) glyceraldehyde 3-phosphate dehydrogenase.

Figure 6: (A) Western blot analysis of TNC, SPARC and OPN protein expression in MSCs grown on gelatin/HA scaffold. GAPDH is the loading control. (B) band densitometry of TNC, SPARC and OPN protein expression. Data represent results from three independent experiments. Each individual assay was performed in triplicate and expressed as mean $\pm \mathrm{SD}$; ${ }^{*} \mathrm{P}<0.05$ as compared to cells grown in monolayer for 28 days (D2). 
with cell viability studies which demonstrated that modification in cell viability are detected in the first week of cell culture [23].

Furthermore, TEM images demonstrated a good cell adhesion on scaffold surface and high extracellular matrix production. All these results clearly showed elevated biocompatibility of the material and its power in inducing in vitro cell adhesion.

The implantation of biomaterials into the body elicits a materialdependent inflammatory response called the foreign body reaction [24]. Macrophages play a pivotal role in the foreign body reaction, orchestrating the pro-inflammatory microenvironment around the biomaterials by secreting cytokines, chemokines and growth factors. When the biomaterial is porous or degradable, such as gelatin-based scaffolds, macrophages can migrate into the material and continue generating a pro-inflammatory microenvironment inside the materials [25].

Matricellular proteins have provided some evidence as having a role in inflammation and foreign body reaction [12]. Since matricellular proteins are involved in inflammation and the foreign body reaction induced by a biomaterial, low expression could be connected to elevated biocompatibility of the material. On the contrary, elevated expression could be related to aberrant homeostasis which leads to aberrant tissue regeneration [12].

Our hypothesis was that elevated expression of the matricellular proteins, such as TNC, SPARC and OPN, in cells grown on implant materials was the key signal for potential encapsulation, foreign body reaction and the inflammatory response which could strongly impair the efficiency of the material.

An high expression of these markers is connected with cell suffering conditions and to simulate in vitro stress conditions, we treated the MSCs with $0.5 \mathrm{mM}$ hydrogen peroxide, a well-known inductor of oxidative stress and apoptosis, for $3 \mathrm{~h}$ [18]. Results showed an high expression of TNC, SPARC and OPN compared to untreated cells.

To assay the expression of TNC, SPARC and OPN a qRT-PCR was carried out. The analysis of matricellular proteins was based on qRTPCR.

qRT-PCR represents a very sensitive method and an improvement in the knowledge regarding the toxicity and biocompatibility of implant materials analyzed according to ISO standard 10993-5, based mainly on viability tests.

Our results demonstrated a low expression of matricellular proteins in cells seeded on gelatin hydroxyapatite scaffolds suggesting that the material utilized in implant conditions could successfully supports normal tissue renewal and counteracts a pro-inflammatory microenviroment. On the contrary, an high expression of TNC, SPARC and OPN was demonstrated in MSCs culture on carboxymethyl cellulose based scaffolds, biomaterial previously demonstrated as potential inductor of encapsulation and foreign body reaction [14].

The expression of TNC mRNA had previously been utilized as a biocompatibility marker in human fibroblasts grown in contact with methacrylate-based material, in which a long term exposition of the cells to the material induced cell suffering and an upregulation of TNC [26].

The qRT-PCR data were confirmed by western blot analysis in which upregulation of TNC preotein was observed after 21 days of culture while upregulation of SPARC and OPN proteins were detected after 21 and 28 days of cell growing on biomaterial.

\section{Conclusion}

We propose the analysis of matricellular proteins as an additional parameter in evaluating the biocompatibility of implant materials in vitro before in vivo testing. We tested the biocompatibility of a gelatin/ HA scaffold, based on the analysis of the expression of TNC, SPARC and OPN.

Transmission electron microscopy analysis was carried out to evaluate the cell growth and morphological changes induced by cell/ scaffold interactions while qRT-PCR and western blot analysis were carried out to quantify gene and protein expression.

qRT-PCR showed a low expression of TNC, SPARC and OPN proteins in cells grown on gelatin/HA scaffolds as compared cells cultured in monolayer, suggesting that low expression of the matricellular proteins in cells grown in contact with implant scaffolds could be connected with a more suitable environment for the material in contrast to encapsulation, foreign body reaction and inflammatory response. Therefore, we hypothesized that this test system has the potential of enhancing the predictability of in vivo behaviour and the biocompatibility of biomimetic materials in inducing adverse reactions after implantation. According to our results, the gelatin/HA scaffold, used in our study as a biomimetic model of a scaffold, is highly biocompatible, suggesting its potential in contrasting adverse reactions after in vivo implantation.

\section{Acknowledgments}

This study was supported by the Italian Ministry of Research and Technology (MURST) with an FIRB grant (RBAP10MLK7_005), PRIN 2009 grant, and a Fondazione del Monte di Bologna and Ravenna 2012 grant.

\section{References}

1. http://www.iso.ch/iso/en/ISOOnline.frontpage 20

2. Fotakis G, Timbrell JA (2006) In vitro cytotoxicity assays: comparison of LDH, neutral red, MTT and protein assay in hepatoma cell lines following exposure to cadmium chloride. Toxicol Lett 160: 171-177.

3. Vogel CF, Garcia J, Wu D, Mitchell DC, Zhang Y, et al. (2012) Activation of inflammatory responses in human U937 macrophages by particulate matter collected from dairy farms: an in vitro expression analysis ofpro-inflammatory markers. Environ Health. 28 11-17.

4. Pizzoferrato A, Ciapetti G, Stea S, Cenni E, Arciola CR, et al. (1994) Cell culture methods for testing biocompatibility. Clin Mater 15: 173-190.

5. Ratner BD (2002) Reducing capsular thickness and enhancing angiogenesis around implant drug release systems. J Control Release 78: 211-218.

6. Kyriakides TR, Bornstein P (2003) Matricellular proteins as modulators of wound healing and the foreign body response. Thromb Haemost 90: 986-992.

7. Frangogiannis NG (2012) Matricellular proteins in cardiac adaptation and disease. Physiol Rev 92: 635-688.

8. Sangaletti S, Colombo MP (2008) Matricellular proteins at the crossroad of inflammation and cancer. Cancer Lett 267: 245-253.

9. Bornstein P (2009) Matricellular proteins: an overview. J Cell Commun Signal 3: $163-165$.

10. Yang Z, Kyriakides TR, Bornstein $P$ (2000) Matricellular proteins as modulators of cell-matrix interactions: adhesive defect in thrombospondin 2-null fibroblasts is a consequence of increased levels of matrix metalloproteinase-2. Mol Biol Cell 11: 3353-3364.

11. Chong HC, Tan CK, Huang RL, Tan NS (2012) Matricellular proteins: a sticky affair with cancers. J Oncol 2012: 351089.

12. Chiodoni C, Colombo MP, Sangaletti S (2010) Matricellular proteins: from homeostasis to inflammation, cancer, and metastasis. Cancer Metastasis Rev 29: $295-307$. 
Citation: Durante S, Teti G, Salvatore V, Focaroli S, Mattioli-Belmonte M, et al. (2014) Matricellular Protein Expression and Cell Ultrastructure as Parameters to Test In Vitro Cytotoxicity of a Biomimetic Scaffold. J Cytol Histol 5: 262. doi:10.4172/2157-7099.1000262

13. Panzavolta S, Torricelli P, Amadori S, Parrilli A, Rubini K, et al. (2013) 3D interconnected porous biomimetic scaffolds: In vitro cell response. J Biomed Mater Res A 101: 3560-3570.

14. Moulonguet I, Arnaud E, Bui P, Plantier F (2013) Foreign body reaction to Radiesse: 2 cases. Am J Dermatopathol 35: e37-40.

15. Dominici M, Le Blanc K, Mueller I, Slaper-Cortenbach I, Marini F, et al (2006) Minimal criteria for defining multipotent mesenchymal stromal cells The Inter. Society for Cellular Therapy position statement. Cytotherapy 8: 315-317.

16. Teti G, Cavallo C, Grigolo B, Giannini S, Facchini A, et al. (2012) Ultrastructural analysis of human bone marrow mesenchymal stem cells during in vitro osteogenesis and chondrogenesis. Microsc Res Tech 75: 596-604.

17. Schönherr E, Witsch-Prehm P, Harrach B, Robenek H, Rauterberg J, et al. (1995) Interaction of biglycan with type I collagen. J Biol Chem 270: 2776-2783.

18. Burattini S, Salucci S, Baldassarri V, Accorsi A, Piatti E, et al. (2013) Antiapoptotic activity of hydroxytyrosol and hydroxytyrosyl laurate. Food Chem Toxicol 55: 248-256.

19. Undale AH, Westendorf JJ, Yaszemski MJ, Khosla S (2009) Mesenchymal stem cells for bone repair and metabolic bone diseases. Mayo Clin Proc 84 : 893-902.
20. Zhang QZ, Nguyen AL, Yu WH, Le AD (2012) Human oral mucosa and gingiva: a unique reservoir for mesenchymal stem cells. J Dent Res 91: 1011-1018.

21. Bose S, Roy M, Bandyopadhyay A (2012) Recent advances in bone tissue engineering scaffolds. Trends Biotechnol 30: 546-554.

22. Ducheyne P, Qiu Q (1999) Bioactive ceramics: the effect of surface reactivity on bone formation and bone cell function. Biomaterials 20: 2287-2303.

23. Riss TL, Moravec RA, Niles AL, Benink HA, Worzella TJ, et al. (2013) Cell Viability Assays. In: Sittampalam GS, Gal-Edd N, Arkin M, Auld D, Austin C, et al. Assay Guidance Manual [Internet]. Bethesda (MD): Eli Lilly \& Company and the National Center for Advancing Translational Sciences.

24. Anderson JM, Rodriguez A, Chang DT (2008) Foreign body reaction to biomaterials. Semin Immunol 20: 86-100.

25. van Putten SM, Ploeger DT, Popa ER, Bank RA (2013) Macrophage phenotypes in the collagen-induced foreign body reaction in rats. Acta Biomater 9: 65026510.

26. Zago M, Teti G, Mazzotti G, Ruggeri A, Breschi L, et al. (2008) Expression of procollagen alpha1 type I and tenascin proteins induced by HEMA in human pulp fibroblasts. Toxicol In Vitro 22: 1153-1159. 